\title{
First-principles study of electronic structure of Er-doped monoclinic $\mathrm{ZrO}_{2}$
}

\author{
Yuki OBUKURO ${ }^{1, \dagger}$, Toru OKAWARA ${ }^{2}$ and Tetsuya OKUYAMA ${ }^{1}$ \\ ${ }^{1}$ Department of Materials System Engineering, National Institute of Technology (NIT), Kurume College, \\ 1-1-1 Komorino, Kurume, Fukuoka 830-8555, Japan \\ ${ }^{2}$ Department of Creative Engineering, Materials Chemistry Course, National Institute of Technology (NIT), Kitakyushu College, \\ 5-20-1 Shii, Kokuraminami-ku, Kitakyushu 802-0985, Japan
}

The electronic structure of Er-doped monoclinic $\mathrm{ZrO}_{2}$ was investigated by the generalized gradient approximation (GGA), GGA plus on-site Coulomb interaction (GGA $+U$ ) calculation, and modified Becke-Johnson (MBJ) exchange potential calculation. In the GGA calculation, the minimum bandgap energy of $\mathrm{ZrO}_{2}$ was estimated to be $3.74 \mathrm{eV}$. The valence band was mainly composed of $\mathrm{O} 2 \mathrm{p}$ states, which strongly hybridized with the $\mathrm{Zr} 4 \mathrm{~d}$ states. The conduction band was composed of $\mathrm{Zr} 4 \mathrm{~d}$ and $\mathrm{O} 2 \mathrm{p}$ states. When an Er atom replaced one of the $\mathrm{Zr}$ atoms, seven-fold $\mathrm{Er} 4 \mathrm{f}$ states appeared in the forbidden gap of $\mathrm{ZrO}_{2}$. Considering the spin-orbit coupling, the energy positions of the seven-fold Er $4 \mathrm{f}$ states in the forbidden gap hardly changed. Based on the GGA $+U$ calculation, the Er $4 \mathrm{f}$ states shifted to the lower energy direction and entered into the valence band of $\mathrm{ZrO}_{2}$ with an increase in the $U$ parameter. In addition, the MBJ calculation gave similar results using a small $U$ parameter in the valence band energy region, while the conduction band region is similar to the GGA $+U$ calculation result with a large $U$ parameter. Based on these results, we concluded that the GGA calculation is the most appropriate to describe the position of Er $4 \mathrm{f}$ states in the bandgap of Er-doped $\mathrm{ZrO}_{2}$ phosphors among the three kinds of calculation methods examined in the present study.

(O2019 The Ceramic Society of Japan. All rights reserved.

Key-words : Oxides, Ab initio calculation, Supercell, Electronic structure

[Received May 28, 2019; Accepted September 22, 2019]

$\mathrm{ZrO}_{2}$ is an ideal host material for producing phosphors doped with trivalent lanthanide ( $\mathrm{Ln})$ elements because it has thermal and chemical stabilities. Many researchers have prepared the $\mathrm{Ln}^{3+}$-doped $\mathrm{ZrO}_{2}$ and investigated its photoluminescence properties; for example, $\mathrm{Ce}^{3+}$ doped by Zhu et al., ${ }^{1)} \mathrm{Sm}^{3+}$ doped by Nayak et al., ${ }^{2)} \mathrm{Yb}^{3+}$ doped by Rosa et $\mathrm{al}^{3)}$ and $\mathrm{Er}^{3+}, \mathrm{Yb}^{3+}$ co-doped by Singh et al. ${ }^{4), 5)}$ To understand the luminescence behavior, it is essential to understand the electronic structure of the solid, especially the energy level of the Ln $4 \mathrm{f}$ states. Dorenbos systematically researched the 4 f energy levels of the $\mathrm{Ln}^{2+} /$ $\mathrm{Ln}^{3+}$ doped into $\mathrm{ZrO}_{2}$ and clarified that the $\mathrm{Ln}^{2+} / \mathrm{Ln}^{3+} 4 \mathrm{f}$ levels locate in the bandgap of $\mathrm{ZrO}_{2}{ }^{6}{ }^{6}$ However, there are only a few theoretical reports about the electronic structure of the $\mathrm{Ln}^{3+}$-doped $\mathrm{ZrO}_{2}$ due to the difficulty of strongly localized $4 \mathrm{f}$ electrons. ${ }^{7)}$

For the density functional theory (DFT), local spin density approximation and generalized gradient approximation (GGA) methods have been generally applied as the exchange-correlation functional to solve the Kohn-Sham equation. ${ }^{8)-10)}$ It is well known that these approaches

\footnotetext{
Corresponding author: Y. Obukuro; E-mail: obukuro@ kurume-nct.ac.jp
}

underestimate the bandgaps of semiconductors and insulators, ${ }^{11), 12)}$ although these approaches provide the structural properties of solid-state materials with a high accuracy. In order to improve the bandgap problem, the GGA plus on-site Coulomb interaction $(\mathrm{GGA}+U)$ proposed by Anisimov et al. ${ }^{13)}$ and modified Becke-Johnson (MBJ) exchange potential proposed by Tran and Blaha ${ }^{14)}$ are used. Moreover, the hybrid Hartree-Fock density functional method and the $G W$ approximation based on the quasi-particle approach have been developed to reproduce the accurate electronic structure of solid-state materials. ${ }^{15), 16)}$ However, these sophisticated methods are still expensive for the large-scale system that the application of the supercell method is indispensable. In contrast, the $\mathrm{GGA}, \mathrm{GGA}+U$, and $\mathrm{MBJ}$ methods are reasonable for interpreting the experimental data of various practical materials with complex structures including many atoms, in view of the computational time and cost.

In this study, we performed a first-principles band calculation for the $\mathrm{Er}^{3+}$-doped $\mathrm{ZrO}_{2}$ with a monoclinic structure to investigate the bandgap energy, valence and conduction band characteristics, and position of the Er 4f states in the bandgap in the framework of the GGA calculation. We also compared the calculation results obtained by the 


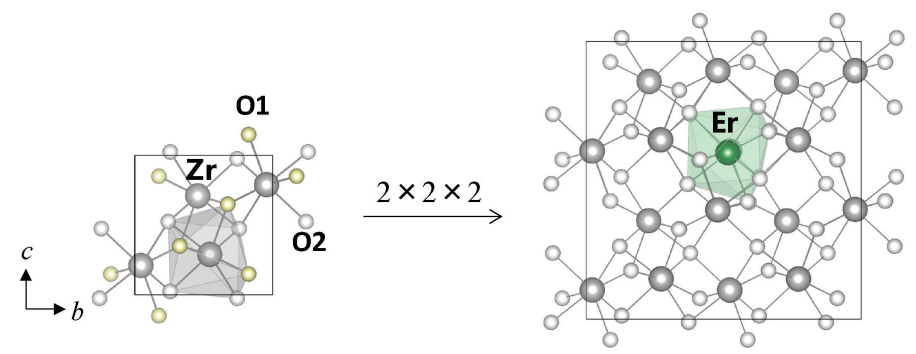

Fig. 1. Schematic drawing of the monoclinic $\mathrm{ZrO}_{2}$ unit cell as well as its extended supercell structure. $\mathrm{Zr}$ (gray), O1 (pale yellow), O2 (white), Er (green).

GGA $+U$ and the MBJ methods with those of the GGA calculation.

Monoclinic $\mathrm{ZrO}_{2}$ was classified into space group $\mathrm{P} 21 / \mathrm{c}$ and contained four formula units for a total of 12 atoms. ${ }^{17)}$ All of the constituent atoms occupy the Wyckoff $4 \mathrm{e}$ sites. An Er-doped $\mathrm{ZrO}_{2}$ supercell was constructed by substituting an Er atom for a $\mathrm{Zr}$ atom in the $2 \times 2 \times 2$ expanded cell (Fig. 1). In this supercell, there were $31 \mathrm{Zr}$ atoms, 64 $\mathrm{O}$ atoms, and one $\mathrm{Er}$ atom. The supercell has the nominal composition of $\mathrm{Er}_{0.03} \mathrm{Zr}_{1.97} \mathrm{O}_{2}$ and the space group $\mathrm{P} 1$. The electronic structure was calculated in two steps. First, the structure optimization calculation was performed in the framework of the GGA method using the CASTEP code. ${ }^{18)}$ The structural parameters of the $\mathrm{ZrO}_{2}$ unit cell $\left(\mathrm{P} 2{ }_{1} / \mathrm{c}\right)$ were optimized by relaxing all lattice constants and atomic positions. For the Er-doped $\mathrm{ZrO}_{2}$ supercell, the lattice constants were assumed to have the same values as those in the expanded cell and only the atomic positions were optimized. The pseudopotentials were constructed using the on-the-fly pseudopotential generator. ${ }^{19}$ ) The cutoff energy was set to $630 \mathrm{eV}$ guaranteeing the convergence of the total energies less than $5.0 \times 10^{-6} \mathrm{eV} /$ atom. The reciprocal space integration over the irreducible Brillouin zone (BZ) was approximated at two irreducible $k$-points using the $2 \times 2 \times 2$ Monkhorst-Pack $k$-point grid. $^{20)}$

Secondly, the all-electron energy band calculations were performed with the full-potential linearized augmented plane wave and local orbital (FLAPW + lo) method based on the GGA approach. ${ }^{21)}$ For the GGA $+U$ calculation, strong correlations among the f electrons were considered through the effective screened interaction among electrons, $U_{\text {eff }}=U-J$, with Coulomb $(U)$ and exchange $(J)$ parameters, which are mean values for the Coulomb and exchange interactions in a considered shell. ${ }^{22}$ For the MBJ calculation, the parameter $c$ was self-consistently determined and $\alpha=-0.012$ and $\beta=1.023$ bohr $^{1 / 2}$ were used. ${ }^{14)}$ The muffin-tin radii $\left(R_{\mathrm{MT}}\right)$ values of the $\mathrm{Er}, \mathrm{Zr}$ and $\mathrm{O}$ atoms for the $\mathrm{Er}^{3+}$-doped $\mathrm{ZrO}_{2}$ supercell were 2.21, 2.00 and 1.81 a.u. (atomic units), respectively. The planewave cutoff was $R_{\mathrm{MT}} \times K_{\max }=6.0$ (5,521 plane waves) for the $\mathrm{Er}^{3+}$-doped $\mathrm{ZrO}_{2}$ supercell. The electroneutral condition in the $\mathrm{Er}^{3+}$-doped supercell was maintained by placing a background charge such as a jellium model. The BZ integration was performed by the modified tetrahedron method within the irreducible wedge. For the valence states, rela-
Table 1. Calculated lattice constants obtained for $\mathrm{ZrO}_{2}$ unit cell and Er-doped supercell. The supercell was obtained by expanding the $\mathrm{ZrO}_{2}$ structure twice each in the directions of the $a-, b$-, and $c$-axes

\begin{tabular}{cccccc}
\hline & & $a / \mathrm{nm}$ & $b / \mathrm{nm}$ & $c / \mathrm{nm}$ & $\beta /^{\circ}$ \\
\hline $\mathrm{ZrO}_{2}$ unit cell & Experimental ${ }^{17)}$ & 0.51974 & 0.52980 & 0.53498 & 99.53 \\
& Calculated $^{2}$ & 0.51849 & 0.52367 & 0.53767 & 99.66 \\
Er-doped supercell & Calculated & 1.03698 & 1.04735 & 1.07534 & 99.66 \\
\hline
\end{tabular}

Table 2. Calculated bond lengths of $\mathrm{Zr}-\mathrm{O}$ in $\mathrm{ZrO}_{2}$ unit cell and Er-O in Er-doped supercell. Seven $\mathrm{O}$ atoms are coordinated around a cation

\begin{tabular}{crlr}
\hline \multicolumn{2}{c}{ Bond length/nm } & \multicolumn{2}{c}{ Bond length/nm } \\
\hline Zr-O1 & 0.2055 & Er-O35 & 0.2142 \\
Zr-O1 & 0.2081 & Er-O57 & 0.2189 \\
Zr-O2 & 0.2164 & Er-O31 & 0.2215 \\
Zr-O1 & 0.2166 & Er-O42 & 0.2250 \\
Zr-O2 & 0.2172 & Er-O16 & 0.2289 \\
Zr-O2 & 0.2271 & Er-O60 & 0.2299 \\
Zr-O2 & 0.2299 & Er-O62 & 0.2438 \\
\hline
\end{tabular}

tivistic effects were included in either a scalar relativistic approximation or a spin-orbit coupling (SOC) that was considered by the second-variation method, while the core states were treated fully relativistically. In the density of states (DOS) calculation, the BZ integration was performed using the modified tetrahedron method on a special mesh of $32 k$ points and $64 k$ points without and with SOC, respectively.

Table 1 lists the optimized lattice constants of the $\mathrm{ZrO}_{2}$ unit cell and the Er-doped $\mathrm{ZrO}_{2}$ supercell. The calculated lattice constants of $\mathrm{ZrO}_{2}$ agreed with the experimental values within $0.24 \%$ for the $a$-axis, $1.16 \%$ for the $b$-axis, $0.50 \%$ for the $c$-axis, and $0.13 \%$ for $\beta$. Table 2 shows the bond lengths of $\mathrm{Zr}-\mathrm{O}$ in the unit cell and the bond lengths of Er-O in the supercell. In the monoclinic $\mathrm{ZrO}_{2}$ structure, seven $\mathrm{O}$ atoms are coordinated around a $\mathrm{Zr}$ atom, and the average bond length of $\mathrm{Zr}-\mathrm{O}$ is $0.2173 \mathrm{~nm}$. Although the coordination number did not change, the bond distance with oxygen increased when the $\mathrm{Zr}$-site was replaced by an Er atom. The average bond length of $\mathrm{Er}-\mathrm{O}$ is $0.2260 \mathrm{~nm}$. This is due to the difference in the ionic radius between $\mathrm{Er}^{3+}(94.5 \mathrm{pm})$ and $\mathrm{Zr}^{4+}(78 \mathrm{pm})$.

Figure 2 shows the calculated band structure and the DOS for the monoclinic $\mathrm{ZrO}_{2}$. The origin of the energy 


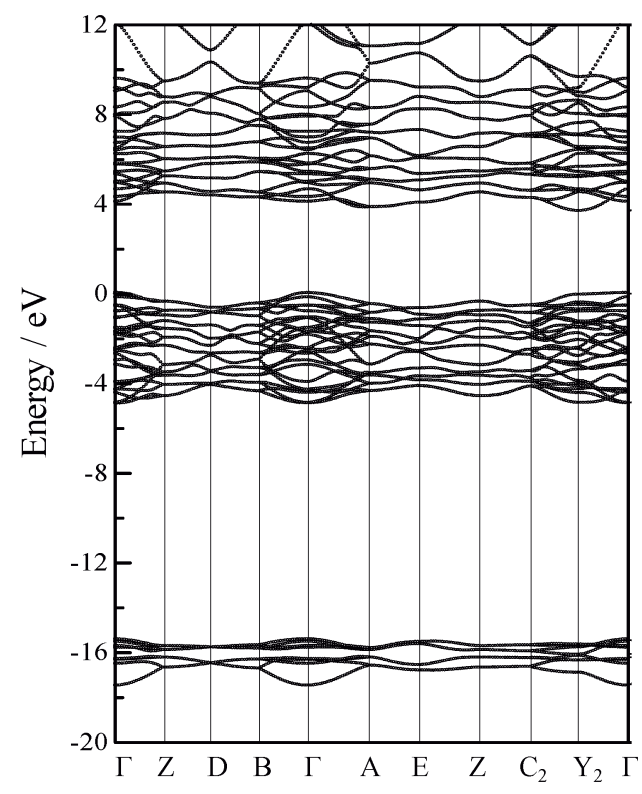

(a)

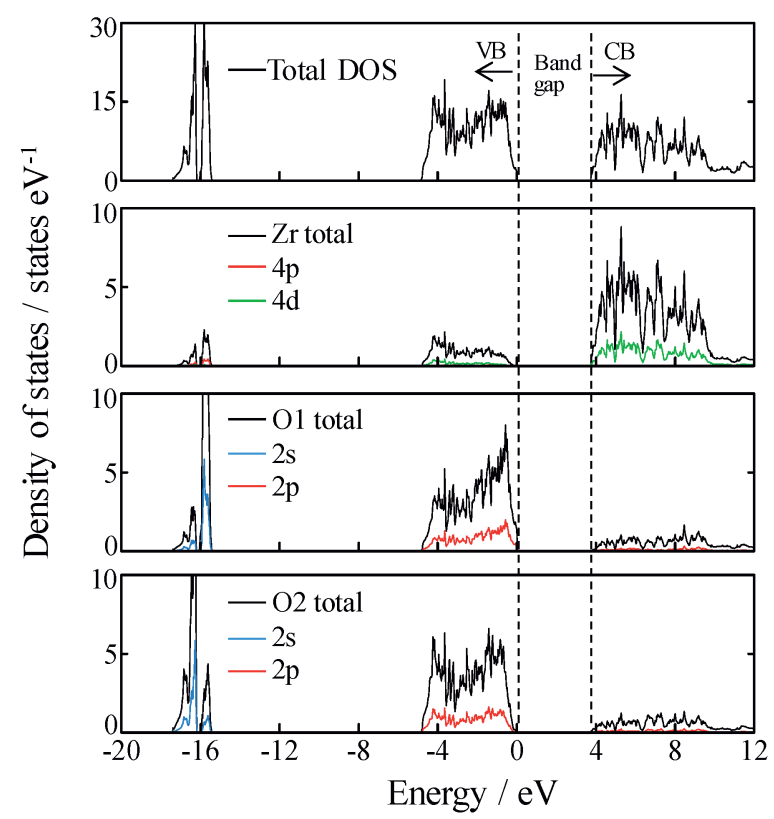

(b)

Fig. 2. Calculated band structure (a) and DOS (b) of monoclinic $\mathrm{ZrO}_{2}$ using the GGA method. The highest occupied energy level with electrons was $0 \mathrm{eV}$. The band path is taken from Ref. 23.

is arbitrarily set as the valence band maximum (VBM). As shown in Fig. 2(a), the VBM and conduction band minimum $(\mathrm{CBM})$ are located at $\Gamma$ and $\mathrm{Y}_{2}$, respectively, indicating that the monoclinic $\mathrm{ZrO}_{2}$ is an indirect-band transition. However, the energy difference between the direct and indirect transitions is very small. The forbidden gap between the VBM and the CBM was $3.74 \mathrm{eV}$, which is smaller than the experimental optical bandgap $(5.5 \mathrm{eV}){ }^{24)}$ This value is due to a lack of consideration for the selfinteraction correction and integer discontinuity in the exchange-correlation energy and potential with a changing number of electrons. ${ }^{11), 12)}$ Figure 2(b) shows the total DOS and the partial DOS for the $\mathrm{Zr}$ and $\mathrm{O}$ atoms. The VB below $-15 \mathrm{eV}$ is constructed from $\mathrm{O} 2 \mathrm{~s}$ and $\mathrm{Zr} 4 \mathrm{p}$ states. The $\mathrm{VB}$ in the energy range of -4.29 to $0 \mathrm{eV}$ is mainly constructed from the $\mathrm{O} 2 \mathrm{p}$ states, which hybridized strongly with $\mathrm{Zr} 4 \mathrm{~d}$ states, indicating stronger covalent bonding. On the other hand, the $\mathrm{CB}$ is mainly composed of $\mathrm{Zr} 4 \mathrm{~d}$ states with a small $\mathrm{O} 2 \mathrm{p}$ contribution.

Figure 3 depicts the total DOS of the Er-doped $\mathrm{ZrO}_{2}$ supercell from the GGA calculation. The forbidden gap of the host $\mathrm{ZrO}_{2}$ was calculated to be $3.51 \mathrm{eV}$. Seven-fold $\mathrm{Er}$ 4f minority-spin states appear in the bandgap of $\mathrm{ZrO}_{2}$ $(-0.27,-0.12,-0.02,0.00,1.15,1.23$ and $1.34 \mathrm{eV})$. This is because there are seven kinds of bond lengths between the $\mathrm{Er}$ and $\mathrm{O}$ atoms as listed in Table 2. These Er $4 \mathrm{f}$ minority-spin states are atomic-like peaks and strongly localized in the gap. In contrast, for the Er 4f majority-spin state, there are no energy states in the bandgap, and the Er 4f majority-spin states overlap with the $\mathrm{O} 2 \mathrm{p}$ states in the VB region. The Er 5d majority-spin and minority-spin states are widely spread in the CB. When the SOC is considered, the Er 4 f majority-spin states also appear in the

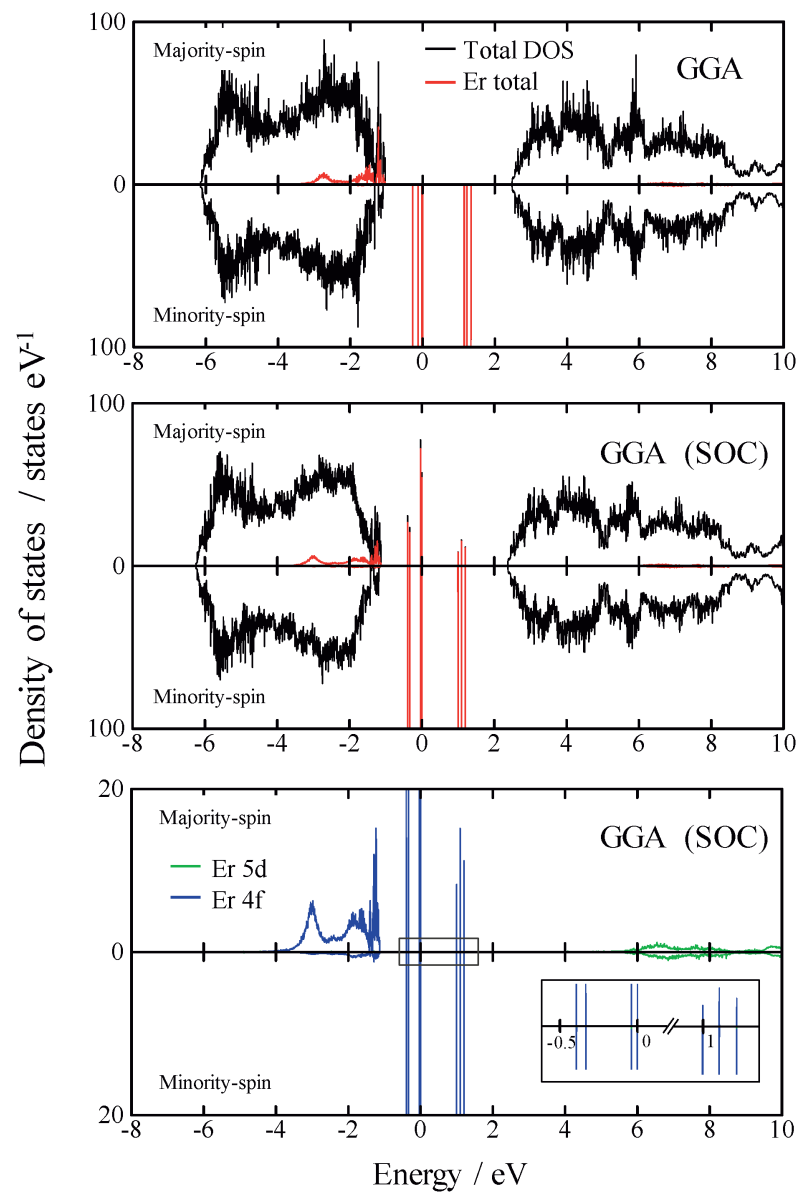

Fig. 3. Density of states of Er-doped $\mathrm{ZrO}_{2}$ supercell calculated from GGA without and with SOC. Sevenfold Er 4f states near $0 \mathrm{eV}$ are inserted. 


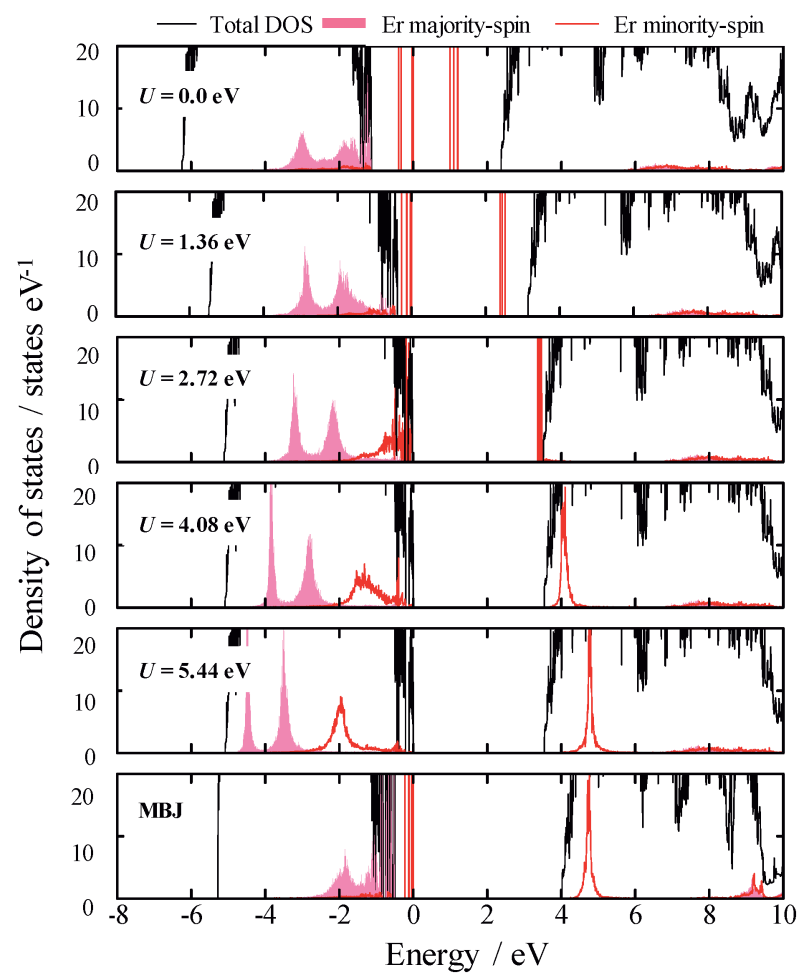

Fig. 4. Density of states of Er-doped $\mathrm{ZrO}_{2}$ calculated from the GGA $+U$ using different $U$ and MBJ methods. The shaded regions illustrate the Er majority-spin character. The red solid lines are the Er minority-spin character.

forbidden gap because the majority-spin and minorityspin states mix with the spin-orbit interaction. However, the energy positions of the seven-fold Er $4 \mathrm{f}$ states in the bandgap of $\mathrm{ZrO}_{2}$ hardly changed (see the inserted figure, at $-0.40,-0.34,-0.04,0.00,1.00,1.09$ and $1.20 \mathrm{eV}$ ).

We then performed the GGA $+U$ calculation using the $U$ parameter for the Er-doped supercell. The $U$ parameter can be generally prepared by fitting to the experimental results. ${ }^{25)-29)}$ However, it is difficult to choose the appropriate screening $U$ parameter, which often changes the obtained results. This means that the position of the Er $4 \mathrm{f}$ states in the bandgap depends on the strength of the Coulomb repulsion. We explored the behavior of the system for the $U$ values varying from $1.36 \mathrm{eV}(0.1 \mathrm{Ry})$ to $5.44 \mathrm{eV}(0.4 \mathrm{Ry}){ }^{30)}$ Figure 4 shows the Er-related DOS calculated from the GGA $+U$ without the SOC. The Er $4 \mathrm{f}$ majority-spin and minority-spin states shifted in the higher energy direction or lower energy direction by considering the $U$ parameter. As the $U$ parameter increases, the Er $4 \mathrm{f}$ minority-spin states strongly shift and enter into the VB and $\mathrm{CB}$ of $\mathrm{ZrO}_{2}$. The Er $4 \mathrm{f}$ minority-spin states also gave a poor dispersibility by adding the $U$ correction in comparison the GGA method. In addition, we noted that the bandgap underestimation for $\mathrm{ZrO}_{2}$ was hardly improved by adding the $U$ parameter $\left(\mathrm{E}_{\mathrm{g}}=3.54 \mathrm{eV} @ U=5.44 \mathrm{eV}\right)$.

According to Dorenbos, ${ }^{6)}$ the $\mathrm{Er}^{3+} 4 \mathrm{f}$ levels locate in the bandgap of the host $\mathrm{ZrO}_{2}$. Although it is a rough interpretation, our result obtained from the GGA calculation showed the tendency suggested by Dorenbos. Similar results are also obtained for $\mathrm{ZrO}_{2}: \mathrm{Yb}^{3+}, \mathrm{Er}^{3+}$ by Pihlgren et al. ${ }^{31)}$ and for $\mathrm{Er}^{3+}$-doped $\mathrm{Y}_{2} \mathrm{O}_{2} \mathrm{~S}$ by Pokhrel et al. ${ }^{32}$ ) In addition, Pihlgren et al. pointed out that the $\mathrm{Er}^{3+} 4 \mathrm{f}$ ground level $\left({ }^{4} I_{15 / 2}\right)$ in $\mathrm{ZrO}_{2}$ locates above the top of the $\mathrm{VB}$ at ca. $5000 \mathrm{~cm}^{-1}(0.62 \mathrm{eV}){ }^{31)}$ According to the information provided by these reports, the above results given by the GGA $+U$ calculation is not suitable to explain the $4 \mathrm{f}$ states of the Er-doped $\mathrm{ZrO}_{2}$ phosphors. Thus, we examined the MBJ exchange potential method. However, as shown in Fig. 4, the MBJ calculation gave similar results using a small $U$ parameter in the VB energy region, while the $\mathrm{CB}$ region is similar to the GGA $+U$ calculation result with a large $U$ parameter. The MBJ calculation was also performed for the Er-doped $\mathrm{ZnO}$, as reported by Alkahtani et al. ${ }^{33)}$ However, the $\mathrm{Er}^{3+} 4 \mathrm{f}$ energy states lie near the bottom of the $\mathrm{CB}$ of $\mathrm{ZnO}$. This result differs from the prediction by Dorenbos, ${ }^{6}$ ) Pihlgren et al., ${ }^{31)}$ and Pokhrel et al. $^{32)}$ as already discussed.

Accordingly, we concluded that the GGA calculation is the most appropriate to describe the behavior of the $\mathrm{Er}^{3+}$ doped $\mathrm{ZrO}_{2}$ phosphors among the three kinds of calculation methods examined at the present time. Needless to say, it should be examined to give a proper treatment to the multiplet effects in the Er 4f energy states unfilled by electrons because the present calculations are essentially based on the one-electron equation in the DFT framework. ${ }^{32)} \mathrm{We}$ hope that the present study concerning electronic structures may be helpful for further experimental study of the $\mathrm{Er}^{3+}$-doped $\mathrm{ZrO}_{2}$ phosphors as a preliminary approach.

Acknowledgements The authors thank Prof. S. Matsushima for his helpful discussion and valuable comments.

\section{References}

1) L. Y. Zhu, X. Q. Wang, G. Yu, X. Q. Hou, G. H. Zhang, J. Sun, X. J. Liu and D. Xu, Mater. Res. Bull., 43, 10321037 (2008).

2) P. K. Nayak, W. J. Kao, D. Sahu and J. L. Huang, J. Am. Ceram. Soc., 93, 3481-3485 (2010).

3) E. de la Rosa, D. Solis, L. A. Diaz-Torres, P. Salas, C. Angeles-Chavez and O. Meza, J. Appl. Phys., 104, 103508 (2008).

4) V. Singh, V. K. Rai and M. Hasse, J. Appl. Phys., 112, 63105 (2012).

5) V. Singh, V. K. Rai, K. Al-Shamery, M. Haase and S. H. Kim, Appl. Phys. A-Mater., 113, 747-753 (2013).

6) P. Dorenbos, J. Luminescense, 151, 224-228 (2014).

7) L. X. Lovisa, J. Andrés, L. Gracia, M. S. Li, C. A. Paskocimas, M. R. D. Bomio, V. D. Araujo, E. Longo and F. V. Motta, J. Alloy. Compd., 695, 3094-3103 (2017).

8) W. Kohn and L. J. Sham, Phys. Rev., 140, A1133A1138 (1965).

9) J. P. Perdew and Y. Wang, Phys. Rev. B, 45, 1324413249 (1992).

10) J. P. Perdew, K. Burke and M. Ernzerhof, Phys. Rev. Lett., 77, 3865-3868 (1996).

11) R. O. Jones and O. Gunnarsson, Rev. Mod. Phys., 61, 689-746 (1989). 
12) P. Mori-Sánchez, A. J. Cohen and W. T. Yang, Phys. Rev. Lett., 100, 146401 (2008).

13) V. I. Anisimov, F. Aryasetiaman and A. I. Lichtenstein, J. Phys. Condens. Matter, 9, 767-808 (1997).

14) F. Tran and P. Blaha, Phys. Rev. Lett., 102, 226401226404 (2009).

15) J. Muscat, A. Wander and N. M. Harrison, Chem. Phys. Lett., 342, 397-401 (2001).

16) H. Jiang, P. Rinke and M. Scheffler, Phys. Rev. B, 86, 125115 (2012).

17) J. E. Jaffe, R. A. Bachorz and M. Gutowski, Phys. Rev. $B, 72,144107$ (2005).

18) V. Milman, B. Winkler, J. A. White, C. J. Pickard, M. C. Payne, E. V. Akhmatskaya and R. H. Nobes, Int. J. Quantum Chem., 77, 895-910 (2000).

19) K. Lejaeghere, V. Van Speybroeck, G. Van Oost and S. Cottenier, Crit. Rev. Solid State Mater. Sci., 39, 1-24 (2014).

20) H. J. Monkhorst and J. D. Pack, Phys. Rev. B, 13, 5188 5192 (1976).

21) P. Blaha, K. Schwarz, G. K. H. Madsen, D. Kvasnicka and J. Luitz, "WIEN2k, An Augmented Plane Wave + Local Orbitals Program for Calculating Crystal Properties", Karlheinz Schwarz, Techn. Universitat Wien, Austria (2001).

22) R. Pentcheva and H. S. Nabi, Phys. Rev. B, 77, 172405 (2008).

23) Y. Hinuma, G. Pizzi, Y. Kumagai, F. Oba and I. Tanaka,
Comput. Mater. Sci., 128, 140-184 (2017).

24) E. Bersch, S. Rangan, R. A. Bartynski, E. Garfunkel and E. Vescovo, Phys. Rev. B, 78, 85114 (2008).

25) S. Matsushima, K. Yamada, H. Nakamura, M. Arai and K. Kobayashi, J. Ceram. Soc. Jpn., 116, 589-592 (2008).

26) J. Holsa, T. Laamanen, M. Lastusaari, J. Niittykoski and P. Novak, J. Rare Earth., 27, 550-554 (2009).

27) K. Obata, Y. Obukuro, S. Matsushima, H. Nakamura, M. Arai and K. Kobayashi, J. Ceram. Soc. Jpn., 121, 766-769 (2013).

28) Y. Obukuro, S. Matsushima, H. Nakamura, M. Arai, H. Yamada and C. N. Xu, Solid State Commun., 186, 4649 (2014).

29) Y. Obukuro, K. Ninomiya, M. Arai, Y. Okuyama, G. Sakai and S. Matsushima, Comput. Mater. Sci., 126, $7-$ 11 (2017).

30) D. Richard, E. L. Munoz, M. Renterı, L. A. Errico, A. Savane and N. E. Christensen, Phys. Rev. B, 88, 165206 (2013).

31) L. Pihlgren, T. Laihinen, L. C. V. Rodrigues, S. Carlson, K. O. Eskola, A. Kotlov, M. Lastusaari, T. Soukka, H. F. Brito and J. Hölsa, Opt. Mater., 36, 1698-1704 (2014).

32) M. Pokhrel, G. A. Kumar, C. G. Ma, M. G. Brik, B. W. Langloss, I. N. Stanton, M. J. Therien, D. K. Sardar and Y. Mao, J. Mater. Chem. C, 3, 11486-11496 (2015).

33) E. A. Alkahtani, A. E. Merad, M. R. Boufatah and A. Benosman, Optik, 128, 274-280 (2017). 